\title{
Management of Disseminated Intravascular Coagulation in Acute Leukemias
}

\author{
Hugo ten Cate ${ }^{1,2}$ Avi Leader $2,3,4$ \\ ${ }^{1}$ Department of Internal Medicine and Thrombosis Expert Center, \\ Maastricht University Medical Center, Maastricht, The Netherlands \\ ${ }^{2}$ CARIM School for Cardiovascular Diseases, Maastricht, \\ The Netherlands \\ ${ }^{3}$ Sackler School of Medicine, Tel Aviv University, Tel Aviv, Israel \\ ${ }^{4}$ Hematology Institute, Rabin Medical Center, Petah Tikva, Israel \\ Hämostaseologie 2021;41:120-126.
}

\begin{abstract}
Address for correspondence Hugo ten Cate, MD, PhD, Departments of Internal Medicine and Biochemistry, Maastricht University Medical Center, UNS50, PO Box 616, 6200 MD Maastricht, The Netherlands (e-mail: h.tencate@maastrichtuniversity.nl).
\end{abstract}

\begin{abstract}
Keywords

- leukemia

- disseminated intravascular coagulation

- management of disease

Disseminated intravascular coagulation (DIC) is characterized by the intravascular activation of coagulation with loss of localization arising from different causes, and is diagnosed using scoring systems which rely upon the presence of an underlying disorder compatible with DIC alongside hemostatic derangements such as low platelet count, prolonged prothrombin time, and elevated fibrinogen degradation products. $\mathrm{DIC}$ is common in patients with acute leukemia, with prevalence ranging from 17 to $100 \%$ in acute promyelocytic leukemia (APL) and 8.5 to $25 \%$ in acute lymphoblastic leukemia (ALL) and non-APL acute myeloid leukemia (AML). The pathophysiology is complex and varies between the leukemia subtypes, and is not fully reflected by the laboratory markers currently used to classify DIC. Similarly, the clinical consequence of DIC in acute leukemia also varies across the types of leukemia. DIC is primarily associated with bleeding in APL, while thrombosis is the dominant phenotype in ALL and non-APL AML. The cornerstone of managing DIC is the treatment of the underlying disease, as exemplified by the important role of early administration of all-trans retinoic acid in APL. Other aspects of management focus on supportive care aimed at minimizing the risk of bleeding, via transfusion of blood products. The use of blood products is more liberal in APL, due to the hemorrhagic phenotype and unacceptably high rates of early hemorrhagic death. This review will focus on the pathophysiology, risk factors, clinical implications, and the management of DIC in patients across the spectrum of acute leukemias.
\end{abstract}

\section{Introduction}

Disseminated intravascular coagulation (DIC) has been defined as "an acquired syndrome characterized by the intravascular activation of coagulation with loss of localization arising from different causes" according to the 1991 ISTH (International Society for Thrombosis and Haemostasis) consensus definition. ${ }^{1}$ DIC always occurs in response to disease, triggered by thromboinflammatory molecules; it can present acutely in relation to sepsis or trauma, or more chronically in association with malignancies, or aortic aneurysm. ${ }^{2}$ A recent inventory among experts listed "acute leukemias" among the high probability diseases associated with DIC. ${ }^{3}$

In a review article on clinical characteristics of DIC in adult patients with either solid tumors or hematologic malignancies, Levi discussed some of the observed heterogeneity in the presenting symptoms and underlying mechanisms of received

December 10, 2020

accepted after revision

February 15, 2021 (c) 2021. Thieme. All rights reserved. Georg Thieme Verlag KG,

Rüdigerstraße 14,

70469 Stuttgart, Germany
DOI https://doi.org/ 10.1055/a-1393-8302. ISSN 0720-9355. 


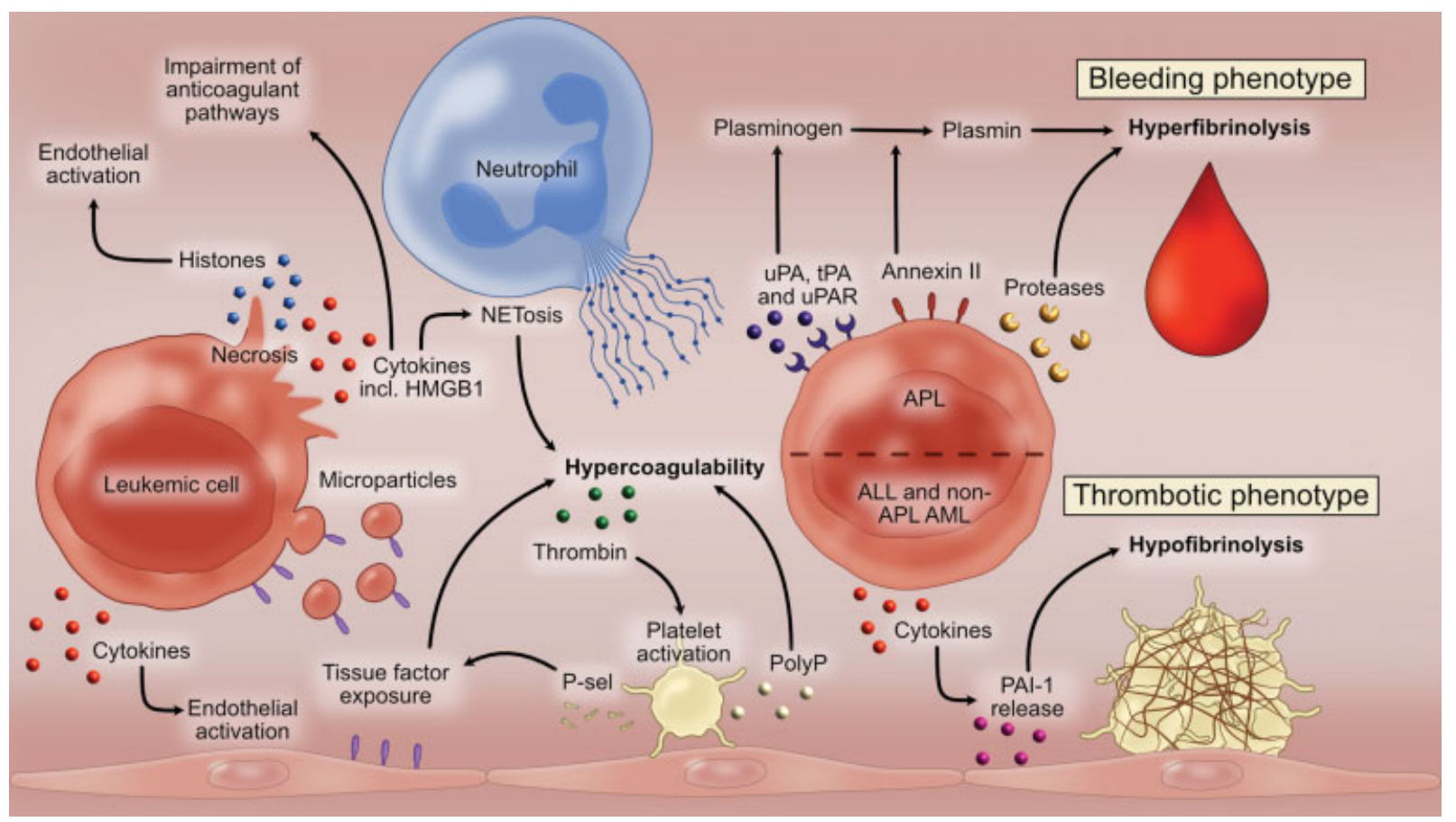

Fig. 1 Leukemic cells share several common procoagulant mechanisms with other tumor cells, including the potential to express tissue factor upon inflammatory stimulation and to liberate extracellular vesicles that further drive coagulation. Apoptosis and cell necrosis, inflicted by chemotherapy may further enhance these effects. Neutrophils are important elements in thromboinflammatory pathways, shedding DNA/histones that interact with platelets as well as factors from the intrinsic cascade to promote thrombin generation. Platelets express and secrete proteins including P-selectin and release polyphosphates (PolyP) that, in concert with neutrophils, catalyze the intrinsic system. Tumor cells in APL have distinct elements including receptors for fibrinolytic proteins urokinase plasminogen activator (uPA) and Annexin II that localize and amplify fibrinolysis, a major contributor to bleeding in APL patients. In other leukemias, fibrinolysis may become impaired by the cytokineinduced increase in plasminogen activator inhibitor 1 (PAI-1) in blood.

DIC, related to the specific coagulopathy ${ }^{4}$ (-Fig. 1); this work also addresses diagnostic challenges associated with the complex syndrome "DIC." To consider a few extremes, acute promyelocytic leukemia (APL)-associated DIC is dominated by bleeding, ${ }^{5}$ while COVID-19-related "DIC" is characterized by thrombosis. ${ }^{6}$ Mechanisms driving these coagulopathies are quite different; however, in both cases the label DIC can be applied if one uses the ISTH DIC score. Although the use of such a simple scoring system cannot probe the complexity of highly different coagulopathies, it remains an accepted starting point in the workup of the patient with DIC, whereby a score of $\geq 5$ points establishes the diagnosis ${ }^{7}$ ( - Fig. 2 and - Table 1). Importantly, in patients not fulfilling this criterion, the score should be repeated for as long as the underlying disease is active, to assess the risks of bleeding and thrombosis related to DIC.

\section{Disseminated Intravascular Coagulation and Acute Leukemia}

Prevalence

The following types of acute leukemias are associated with DIC: acute lymphoblastic leukemia (ALL), non-APL acute myeloid leukemia (AML), and APL. ${ }^{8}$ The prevalence of DIC is highly variable in studies, partially due to the type of leukemia and different patient populations, and ranges from 8.5 to $25 \%$ of patients with non-APL AML or ALL, with another $\pm 15 \%$ of patients also developing DIC soon after the initiation of chemotherapy. ${ }^{9-12}$ In children, DIC has been reported in $14 \%$ of cases of AML, in 3 to $14 \%$ in ALL, and between 17 and $100 \%$ in APL (based on a systematic review by Kongstad et $\mathrm{al}^{13}$ ). Indeed, in APL, DIC is highly prevalent, with up to $90 \%$ of cases fulfilling DIC criteria. ${ }^{13}$

\section{Clinical Presentation and Associated Variables}

The degree of coagulopathy appears to depend in part on the cytogenetic background in relation to the underlying diseases; a normal karyotype was identified as risk factor for the occurrence of DIC in non-APL-type AML. ${ }^{11}$ In such patients, FMS-like tyrosine kinase 3 internal tandem duplication (FLT3-ITD) and nucleophosmin 1 (NMP1) status were independently associated with DIC in the multivariate analysis. ${ }^{9}$ The mechanisms linking these mutations with determinants of DIC still need to be established. The clinical presentation is variable: hemorrhagic manifestations, including life-threatening bleeding, dominate in the majority of studies, but thromboembolic complications also occur frequently. In adult patients with ALL, thrombosis is largely associated with induction therapy, especially in regimens containing L-asparaginase. ${ }^{14}$ However, at the time of diagnosis, baseline D-dimer levels were associated with incident thrombosis, both VTE and arterial thrombosis. The cumulative 100 days of incidence of thrombosis was $53 \%$ in those with al high Ddimer $(\geq 4 \mu \mathrm{g} / \mathrm{mL})$ at baseline versus $14 \%$ in those with 


\section{International Society for Thrombosis and Hemostasis (ISTH) criteria for disseminated intravascular coagulation (DIC)}

1. Risk assessment: Does the patient have an underlying disorder known to be associated with overt DIC? NO: don't use this algorithm

YES:

2. Order global coagulation tests (platelet count, prothrombin time (PT), fibrinogen, soluble fibrin monomers, or fibrin degradation products

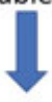

3. Score global coagulation test results:

- Platelet count

- Evelated fibrin-related marker (eg soluble fibrin monomers/fibrin degradation products, eg d-dimer)

- Prolonged prothrombin time

- Fibrinogen level

$(\geq 100=0,<100=1,<50=2)$

(no increase $=0$, moderate increase $=2$, strong increase $=3$ ) $*, * *$ ( $<3$ sec $=0, \geq 3$ but $\leq 6$ sec $=1,>6$ sec $=2$ ) $(\geq 1.0 \mathrm{~g} / \mathrm{L}=0,<1.0 \mathrm{~g} / \mathrm{L}=1)$

4. Calculate score

If > 5: compatible with overt DIC: repeat scoring daily

If $<5$ : suggestive (not affirmative) for non-overt DIC: repeat next 1-2 days

Fig. 2 International Society for Thrombosis and Hemostasis (ISTH) criteria for disseminated intravascular coagulation (DIC). *Based on original ISTH score and suggested modifications (see Suzuki K, Wada H, Imai H, Iba T, Thachil J, Toh CH; Subcommittee on Disseminated Intravascular Coagulation. A re-evaluation of the D-dimer cut-off value for making a diagnosis according to the ISTH overt-DIC diagnostic criteria: communication from the SSC of the ISTH. J Thromb Haemost 2018;16(7):1442-1444).

Table 1 Cut-off values for different commercial D-dimer assays

\begin{tabular}{|l|l|l|}
\hline & 2 points & 3 points \\
\hline STA-Liatest D-Di $(\mu \mathrm{g} / \mathrm{mL})$ & 3.5 & 11.1 \\
\hline Nanopia-D-dimer $(\mu \mathrm{g} / \mathrm{mL})$ & 5.6 & 16.8 \\
\hline LPIA-ACE D-Dimer II $(\mu \mathrm{g} / \mathrm{mL})$ & 6.0 & 20.6 \\
\hline Liasauto D-dimer $(\mu \mathrm{g} / \mathrm{mL})$ & 6.0 & 24.0 \\
\hline Innovance D-dimer $(\mu \mathrm{g} / \mathrm{mL})$ & 4.0 & 13.0 \\
\hline VIDAS D-Dimer Exclusion II $(\mu \mathrm{g} / \mathrm{mL})$ & 3.0 & 7.0 \\
\hline Recommend cut-off values $(\mu \mathrm{g} / \mathrm{mL})$ & 3.0 & 7.0 \\
\hline
\end{tabular}

Source: Suzuki K, Wada H, Imai H, Iba T, Thachil J, Toh CH; Subcommittee on Disseminated Intravascular Coagulation. A re-evaluation of the D-dimer cut-off value for making a diagnosis according to the ISTH overt-DIC diagnostic criteria: communication from the SSC of the ISTH. J Thromb Haemost 2018;16(7):1442-1444.

low/moderate D-dimer levels $(<4 \mu \mathrm{g} / \mathrm{mL})$ in this retrospective study..$^{15}$ Clinically relevant bleeding occurred in $8 \%$ of patients and was not related to D-dimer level.

A similar association between DIC markers and thrombosis has also been shown in AML. In a prospective cohort study of 272 patients with non-APL AML markers of DIC (D-dimer, prothrombin time $[\mathrm{PT}]$, fibrinogen, $\alpha_{2}$-antiplasmin, antithrombin, and platelet count) were assessed before the start of chemotherapy. ${ }^{12}$ Twenty-three (8.5\%) patients had overt DIC (defined as DIC score $\geq 5$ ) at presentation; this number was $8(6.3 \%)$ in the validation cohort comprising 135 newly diagnosed patients older than 60 years. There was no apparent difference regarding AML cytogenetic features among patients with and without DIC $(p=0.25)$. The AMLFAB types correlated with DIC risk ( $p=0.003$ ); in particular, DIC was more frequent in the case of AML of FAB type M5: 12 of 46 patients with an AML M5 classification (26\%) presented with DIC compared with 11 of 224 patients with other FAB types (4.9\%). The association of FAB type M5 and DIC was not statistically significant in the validation cohort. Furthermore, mean white blood cell and blast count in bone marrow were significantly higher in patients with DIC ( $p=0.005$ and $p<0.001$, respectively). Elevated D-dimer levels were strongly associated with thrombosis with hazard risks of 12.3 in the first cohort and 7.8 in the validation cohort at a cumulative incidence of venous and arterial thrombosis of approximately $10 \%$ (8.7\% in the younger cohort, $10.4 \%$ in the elderly patients). This risk already existed at baseline prior to the initiation of therapy. ${ }^{12}$

\section{Pathophysiology}

In acute leukemias, many procoagulant mechanisms have been identified including expression of tissue factor and other procoagulant molecules at leukemic cells and on circulating extracellular vesicles (EVs). The release of cytokines and other mediators may trigger platelets toward a "super activated state" that accelerates coagulation. ${ }^{14}$ These systems may be boosted by chemotherapy-associated massive cell death, but also through drug-specific effects including lowering of antithrombin in case of L-asparaginase. A related phenomenon is the liberation of intracellular histones as part of neutrophil extracellular traps (NETs) and high 
mobility group box 1 (HMGB1); this process is known to trigger coagulation in a neutrophil, platelet, and contact factor-accelerated fashion. NET secretion is one of the postulated mechanisms that, in parallel with platelet-secreted polyphosphates, trigger the coagulation system via direct interactions with the contact factors, providing polyanionic, calcium-rich, phospholipid surfaces to drive coagulation activation. ${ }^{16}$ Indeed, in patients with acute leukemias, levels of HMGB1 and histone 3 were elevated in those with DIC, as compared with non-DIC subjects. ${ }^{17}$

Although in APL similar thromboinflammatory mechanisms are operational, APL also has certain distinctly different features as the leukemic cells express not only tissue factor but also annexin 2 that binds and activates plasminogen, which together with a lower plasma level of thrombin-activatable fibrinolytic inhibitor aggravates fibrinolysis. ${ }^{18}$ These factors likely contribute to the high bleeding risk in APL.

In APL, risk factors for bleeding are hypofibrinogenemia, an ISTH/DIC score $\geq 6$, leukocytosis $>20 \times 10^{9} / \mathrm{L}$, peripheral blasts, reduced renal function, and general poor health. A review of risk factors, derived from mostly retrospective studies, shows that peripheral blood disease burden, characterized by total white blood cell count or circulating blast count greater than $10 \times 10^{9} / \mathrm{L}$, was best predictors of major bleeding in 12 of 15 studies in APL patients. ${ }^{19}$ Remarkably, markers of DIC were inconsistently associated with bleeding; platelet count and low fibrinogen were related only with bleeding in 4 and 7 out of 15 studies, respectively. ${ }^{19}$ In a large cohort of APL patients, high whole white blood cell count $(\geq 20000 / \mathrm{mL}$ ) was an independent predictor for hemorrhagic death; none of the DIC parameters had any significant predictive value. ${ }^{20} \mathrm{~A}$ possible cause of the limited diagnostic utility of DIC scoring to predict bleeding is that only circulating factors are probed, while cellular interactions between malignant cells, platelets, and dysfunctional or inflamed endothelium may be more relevant for bleeding. A second issue is that patients in whom APL is diagnosed, upon presentation with a bleeding diathesis, are rapidly transfused with platelets, plasma, and fibrinogen, which may mask underlying deficiencies and obviously affects bleeding risk; in contrast, increased leukocyte counts will take several days to decline. ${ }^{19}$ Importantly, in this study, thrombocytopenia became clinically relevant only when reaching very low levels, $<5 \times 10^{9} / \mathrm{L}$, rare in APL; moreover, the bleeding risk in the settings of similarly severe thrombocytopenia differs in APL as compared with non-APL AML, suggesting that other factors rather than platelet count are important in APL-related bleeding.

Both arterial and venous thromboembolisms have also been reported in APL in 0.9 to $15 \%$ of cases at presentation. Risk factors for thrombosis are leukocytosis, FLT3-ICD mutations, CD2 expression, and CD15 expression. ${ }^{21,22}$

\section{Management of Disseminated Intravascular Coagulation}

General

As stated, DIC is an indicator of severe disease and, in general, it will subside only when the underlying disease is under control, meaning that adequate treatment of leukemia is key in reversing coagulopathy.

On top of the prevalent risk factors for thrombosis and bleeding in acute leukemias, treatment may also be complicated by thromboembolic complications. A specific challenge is to control coagulopathy associated with chemotherapy or specific treatments like CD-19-targeted chimeric antigen receptor $\mathrm{T}$ cell (CAR-T) and the associated cytokine release syndrome, in cases of relapsed and refractory B-ALL in which coagulopathy (up to $50 \%$ of patients) and DIC are frequent phenomena. ${ }^{23,24}$ In their series of 51 patients, Wang et al observed a high rate of clinically relevant bleeding events (10/51), for which plasma products were administered in case of prolonged clotting times, platelets transfused to maintain a platelet count greater than $20 \times 10^{9} / \mathrm{L}$, all interventions without overt thrombotic complications. ${ }^{24}$

In general, a key issue in the management of acute leukemia-associated DIC is to limit the risk of life-threatening bleeding. Hence, vigilance for DIC on a daily basis is critically important, in particular related to platelet counts less than 10 to $20 \times 10^{9} / \mathrm{L}$ that should trigger platelet transfusion in the absence of overt bleeding. In case of active bleeding, the threshold for platelet transfusion is set at less than $50 \times 10^{9} / \mathrm{L}$ in the recent ISTH guidance report. ${ }^{25}$ Management of DIC without overt bleeding in acute leukemia differs between APL and the other acute leukemias, especially with respect to transfusion thresholds, whereby a more aggressive transfusion strategy is utilized in APL. ${ }^{25}$

\section{Acute Lymphoblastic Leukemia and Asparaginase}

In ALL patients receiving L-asparaginase (L-ASP), it is important to differentiate between DIC associated with ALL and LASP-associated coagulopathy which is a distinct entity associated with an increased risk of thrombosis and bleeding. LASP depletes asparagine in lymphoid leukemic cells, but it also deaminates circulating glutamine to glutamic acid and its depletion has impacted both on platelet function and on clot-forming properties. ${ }^{26-28}$ Finally, L-ASP in combination with steroids can suppress the natural anticoagulants, antithrombin and plasminogen, thus amplifying thrombin generation. ${ }^{29}$ Since L-ASP results in hypofibrinogenemia and prolonged PT, differentiating it from DIC may be challenging. The management of this entity differs from that of DIC, as detailed in recent guidelines. ${ }^{30}$ One important component is monitoring antithrombin levels which may become critically reduced and require suppletion with antithrombin concentrate at levels as low as 50 to $60 \%{ }^{30}$ The SSC guidance document also suggests correction of fibrinogen levels less than $0.5 \mathrm{~g} / \mathrm{L}$, aiming at higher levels in those with active bleeding. ${ }^{25}$ In addition, low-molecular-weight heparin prophylaxis is suggested during L-ASP induction therapy, unless there is severe thrombocytopenia or otherwise a high bleeding risk. ${ }^{25,30}$

Acute Promyelocytic Leukemia and All-Trans Retinoic Acid Before the introduction of all-trans retinoic acid (ATRA), bleeding tendency in APL was profound and life threatening with intracranial bleeding and pulmonary hemorrhage in up 
to $56 \%$ of cases. Since the introduction of ATRA, the outcome of patients has markedly improved. ATRA has a strong effect on APL-associated coagulopathy with reduction in prothrombotic markers within 4 to 8 days. ${ }^{5,19}$ In particular, ATRA reverses coagulopathy faster than it reduces circulating leukemic cell burden, suggesting additional beneficial effects of this agent on the inflammasome and/or the expression of procoagulant molecules on the APL cells. ${ }^{31}$ One effect of major importance was the identification of the downregulation of tissue factor and concurrent upregulation of thrombomodulin (TM) in APL cells treated with ATRA. ${ }^{32}$ Comparably, leukemic cells from AML patients were similarly modified toward a more anticoagulant profile using retinoids. $^{33}$

However, in spite of reduced mortality, the risk of major and fatal bleeding remains very high: 3 to $4.5 \%$ before the start of ATRA, but persistently elevated during the first weeks of ATRA treatment. Unfortunately, early death due to coagulopathy and bleeding still is "the foremost obstacle to remission induction" 19 occurring in approximately $5 \%$ of all APL patients in prospective trials, ${ }^{34}$ with higher figures for hemorrhage-related deaths reported in cohort studies (up to 13\%; summarized in Kwaan et $\mathrm{al}^{18}$ ). The combination of ATRA with arsenic trioxide (ATO) may further diminish the risk of major hemorrhage as suggested by recent trials with this combination. ${ }^{35,36}$ Accordingly, a dose of ATRA is administered the moment a high clinical suspicion of APL arises, even before the diagnosis is confirmed by bone barrow aspiration and molecular tests.

For the aforementioned reasons, platelet counts and coagulation tests including fibrinogen levels and fibrin cleavage products should be measured daily. To limit the risk of bleeding, transfusions of fibrinogen and/or cryoprecipitate, platelets, and fresh frozen plasma are warranted to maintain a fibrinogen concentration above 100 to $150 \mathrm{mg} / \mathrm{dL}$, the platelet count above 30 to $50 \times 10^{9} / \mathrm{L}$ (or a threshold $20-30 \times 10^{9} / \mathrm{L}$ according to ISTH guidance document ${ }^{25}$ ), and the INR below 1.5 , to be continued until resolution of coagulopathy. ${ }^{37}$

Although initial small studies suggested that the use of tranexamic acid, as antifibrinolytic therapy, would be helpful in restoring excess fibrinolysis and bleeding risk, the largest retrospective study to date did not show any statistically relevant effect on outcomes in APL patients. ${ }^{38}$ Antifibrinolytic therapy with tranexamic acid was used in one study in children with APL. ${ }^{13}$ The use of tranexamic acid is still subject of several randomized controlled trial (RCTs) in patients with hematologic malignancies, as its application is suggested by the Scientific Sub Committee (SSC) guidance proposal as adjuvant therapy for patients with severe thrombocytopenia and refractory to platelet transfusion. ${ }^{25}$

The use of recombinant factor VIIa, which has a certain place in the management of bleeding in patients with hemophilia, has not yet proven to be beneficial in APL. ${ }^{19}$

In spite of the benefits on the combination therapy, ATRA alone, and also in combination with ATO, has several prothrombotic effects that may shift the balance toward overt thrombosis, including endothelial cell activation, accelerated

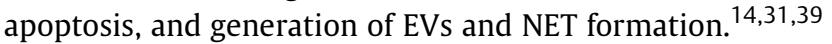

In spite of these prothrombotic effects, heparin is not routinely given as thromboprophylaxis because the risk of lifethreatening bleeding outweighs the risk of thrombosis in patients with APL. ${ }^{25}$ The use of thromboprophylaxis with heparin or low-molecular-weight heparin to prevent or limit DIC is conceptually attractive; however, its use is associated with an increased risk of bleeding. Hence, and particularly in patients with thrombocytopenia, a population in whom the combination of any anticoagulant is very challenging, ${ }^{40}$ the use of heparin prophylaxis is not generally recommended. Also in APL, the previous practice of using IV heparin ${ }^{41}$ has been discontinued due to increased transfusion requirements and risk of delayed bleeding in the absence of any beneficial effects. $^{38,42}$

Recombinant soluble thrombomodulin (recTM) is a compound that has been extensively studied in Japan, for DIC associated with sepsis, and also in several smaller studies and one larger postmarketing study in APL. TM is a cellular receptor for thrombin prevalent on endothelial cells and several other cells. On the vascular endothelium, TM is a critical mediator of the thrombin-catalyzed activation of protein C, a natural anticoagulant and anti-inflammatory protease. Through its lectin domain, TM also directly displays anti-inflammatory properties, including cleavage of HMGB1. The recombinant, soluble form of TM (comprising the extracellular domain of TM) is thought to primarily affect thromboinflammation through the neutralization of several damage-associated molecular patterns, including histones and HMGB1, as well as activated complement factors. ${ }^{43}$ Reversal of laboratory indices of DIC during treatment with recTM was observed, as well as a reduction in hemorrhagic death. ${ }^{44}$ However, DIC in sepsis is different from leukemias in the levels of protein C, and it may be difficult to predict the risk of bleeding related to recTM infusion and generation of activated protein $\mathrm{C}$, in the latter patients. Larger appropriately focused clinical trials in acute leukemias would be needed to assess the value of recTM for these indications. In an updated Cochrane review of interventions aimed at DIC in (subgroups of) acute (and chronic) leukemia patients in RCTs, the authors concluded that the available evidence remains of very low quality and not in favor of any of the following interventions: human activated protein C, recombinant human soluble TM, or dermatan sulfate. ${ }^{45}$

Would there be novel, more promising anticoagulants to counteract DIC in patients with acute leukemias? Obviously, the choice is limited by the relatively high risks of bleeding complications, in particular in those with low platelet counts and/or low fibrinogen levels. Currently, inhibitors of factor XI (a) are being explored in phase 2 clinical trials for their efficacy and safety with regard to prevention of VTE or stroke in patients with arterial vascular disease or atrial fibrillation. ${ }^{46}$ The premise with this type of intervention is effective inhibition of the coagulation system but less impairment of hemostasis, based on the observation that patients who lack factor XI have a rather mild bleeding phenotype. For comparable reasons, inhibitors of factor XII or kallikrein (PKa) could be considered and some are indeed being tested in clinical studies. Although promising from an experimental 
perspective (thrombosis prevention, no increased risk of bleeding), it needs to be established whether inhibiting the contact factor system, in general, comes without unexpected side effects, since PKa and factor XIla are directly connected with inflammation through generation of kallikrein and bradykinin, important vascular mediators. Contact activation may also affect fibrinolysis and such effects could be critical in conditions like APL. Inhibiting factor XIa probably provides a safer strategy, as it is somewhat disconnected from the bradykinin-kallikrein axis, acting primarily in a procoagulant direction.

\section{Conclusions}

A diagnosis of DIC based on consensus criteria remains a sign of poor prognosis in a patient with any disease. A substantial proportion of patients with acute leukemias encounter DIC associated with tumor burden and other risk factors. Treatment of leukemia, when effective, also corrects DIC, although, in particular in APL, immediate treatment with combination therapy still takes time to correct the bleeding diathesis as primary manifestation of DIC. There are no known interventions that specifically correct DIC in leukemia (or other diseases) without harm. Heparin increases the risk of bleeding and for that matter it is relatively contraindicated in these patients. Soluble recTM, while having some potential in sepsis-associated DIC, is not an established therapy in leukemia. Theoretically, other targets in the coagulation system may be explored. Recent developments include agents that inhibit components of the contact activation system including factor XIIa and kallikrein, or factor XIa as a next step in the intrinsic route. ${ }^{47}$ Potentially, these provide safer anticoagulants with less negative impact on hemostasis. Clinical trials with factor XIa inhibitors are ongoing for other indications and these studies need to reveal whether any gain in the efficacy:safety ratio can be obtained. Clinical trials with any anticoagulant in patients at risk for or with overt DIC remain extremely challenging for pharmaceutical companies, certainly in patients with leukemia and additional risks including thrombocytopenia and other risk factors for bleeding. Other targets that relate to the coagulation system including the inflammasome, NETosis, and complement activation may provide additional ways to modify the course of DIC in acute leukemia patients, but on top of better mechanistic insight, courage will be needed to translate new insight into better management.

\section{Conflict of Interest}

$\mathrm{HtC}$ received research funding from Bayer and he is consultant for Alveron.

\section{Acknowledgments}

Ht.C. is supported by grants from CVON RACE 5 and CONTRAST consortia, the REG MED XB Moonshot program, and he is an adjunct professor with the Center for Thrombosis and Hemostasis, Gutenberg University, Mainz, Germany. Aaron Iding, MD, prepared the figure.

\section{References}

1 Taylor FB Jr, Toh CH, Hoots WK, Wada H, Levi M. Scientific Subcommittee on Disseminated Intravascular Coagulation (DIC) of the International Society on Thrombosis and Haemostasis (ISTH) Towards definition, clinical and laboratory criteria, and a scoring system for disseminated intravascular coagulation. Thromb Haemost 2001;86(05):1327-1330

2 Mandernach MM, Kitchens CS. Disseminated intravascular coagulation. In: Kitchens CS, Kessler CM, Konkle BA, Streif MB, Garcia DA. Consultative Hemostasis and Thrombosis. 4th ed. Elsevier; 2019:207-226

3 Squizzato A, Gallo A, Levi M, et al. Underlying disorders of disseminated intravascular coagulation: communication from the ISTH SSC Subcommittees on Disseminated Intravascular Coagulation and Perioperative and Critical Care Thrombosis and Hemostasis. J Thromb Haemost 2020;18(09):2400-2407

4 Levi M. Clinical characteristics of disseminated intravascular coagulation in patients with solid and hematological cancers. Thromb Res 2018;164(Suppl 1):S77-S81

5 Thomas X. Acute promyelocytic leukemia: a history over 60 years -from the most malignant to the most curable form of acute leukemia. Oncol Ther 2019;7(01):33-65

6 Iba T, Levy JH, Connors JM, Warkentin TE, Thachil J, Levi M. The unique characteristics of COVID-19 coagulopathy. Crit Care 2020; 24(01):360

7 Levi M, Scully M. How I treat disseminated intravascular coagulation. Blood 2018;131(08):845-854

8 Franchini M, Di Minno MN, Coppola A. Disseminated intravascular coagulation in hematologic malignancies. Semin Thromb Hemost 2010;36(04):388-403

9 Guo Z, Chen X, Tan Y, Xu Z, Xu L. Coagulopathy in cytogenetically and molecularly distinct acute leukemias at diagnosis: comprehensive study. Blood Cells Mol Dis 2020;81:102393

10 Barbui T, Falanga A. Disseminated intravascular coagulation in acute leukemia. Semin Thromb Hemost 2001;27(06):593-604

11 Uchiumi H, Matsushima T, Yamane A, et al. Prevalence and clinical characteristics of acute myeloid leukemia associated with disseminated intravascular coagulation. Int J Hematol 2007;86(02):137-142

12 Libourel EJ, Klerk CPW, van Norden Y, et al. Disseminated intravascular coagulation at diagnosis is a strong predictor for thrombosis in acute myeloid leukemia. Blood 2016;128(14):1854-1861

13 Kongstad C, Mikkelsen TS, Hvas AM. Disseminated intravascular coagulation in children with cancer: a systematic review. Pediatr Hematol Oncol 2020;37(05):390-411

14 Del Principe MI, Del Principe D, Venditti A. Thrombosis in adult patients with acute leukemia. Curr Opin Oncol 2017;29(06): 448-454

15 Anderson DR, Leader A, Karrison TG, Stock A. D-dimer as a predictor of thrombotic events during early acute lymphoblastic leukemia therapy. Blood 2020;136(Suppl 1):5-6. Doi: 10.1182blood-2020140324

16 Rangaswamy C, Englert H, Deppermann C, Renné T Polyanions in coagulation and thrombosis: focus on polyphosphate and NETs. Thromb Haemost 2020; ( epub ahead of print). Doi: 10.1055/a1336-0526

17 Harada-Shirado K, Wang X, Mori H, et al. Circulating intranuclear proteins may play a role in development of disseminated intravascular coagulation in individuals with acute leukemia. Int J Hematol 2020;111(03):378-387

18 Kwaan HC, Weiss I, Tallman MS. The role of abnormal hemostasis and fibrinolysis in morbidity and mortality of acute promyelocytic leukemia. Semin Thromb Hemost 2019;45(06):612-621

19 Naymagon L, Mascarenhas J. Hemorrhage in acute promyelocytic leukemia: Can it be predicted and prevented? Leuk Res 2020; 94:106356

20 Mantha S, Goldman DA, Devlin SM, et al. Determinants of fatal bleeding during induction therapy for acute promyelocytic leukemia in the ATRA era. Blood 2017;129(13):1763-1767 
21 Mitrovic M, Suvajdzic N, Elezovic I, et al. Thrombotic events in acute promyelocytic leukemia. Thromb Res 2015;135(04): 588-593

22 de Albuquerque Antunes A, Breviglieri CNM, Celeste DM, Garanito MP, Cristofani LM, Carneiro JDA. Prevalence and outcomes of thrombotic and hemorrhagic complications in pediatric acute promyelocytic leukemia in a tertiary Brazilian center. Hematol Transfus Cell Ther 2020; (epub ahead of print) . Doi: 10.1016/j. htct.2020.06.017

23 Jiang H, Liu L, Guo T, et al. Improving the safety of CAR-T cell therapy by controlling CRS-related coagulopathy. Ann Hematol 2019;98(07):1721-1732

24 Wang Y, Qi K, Cheng H, et al. Coagulation disorders after chimeric antigen receptor $T$ cell therapy: analysis of 100 patients with relapsed and refractory hematologic malignancies. Biol Blood Marrow Transplant 2020;26(05):865-875

25 Wang TF, Makar RS, Antic D, et al. Management of hemostatic complications in acute leukemia: guidance from the SSC of the ISTH. J Thromb Haemost 2020;18(12):3174-3183

26 Zakarija A, Kwaan HC. Adverse effects on hemostatic function of drugs used in hematologic malignancies. Semin Thromb Hemost 2007;33(04):355-364

27 Goyal G, Bhatt VR. L-asparaginase and venous thromboembolism in acute lymphocytic leukemia. Future Oncol 2015;11(17): 2459-2470

28 Hua VM, Abeynaike L, Glaros E, et al. Necrotic platelets provide a procoagulant surface during thrombosis. Blood 2015;126(26): 2852-2862

29 Truelove E, Fielding AK, Hunt BJ. The coagulopathy and thrombotic risk associated with L-asparaginase treatment in adults with acute lymphoblastic leukaemia. Leukemia 2013;27(03):553-559

30 Zwicker JI, Wang TF, DeAngelo DJ, et al. The prevention and management of asparaginase-related venous thromboembolism in adults: guidance from the SSC on hemostasis and malignancy of the ISTH. J Thromb Haemost 2020;18(02):278-284

31 David S, Mathews V. Mechanisms and management of coagulopathy in acute promyelocytic leukemia. Thromb Res 2018;164 (Suppl 1):S82-S88

32 Koyama T, Hirosawa S, Kawamata N, Tohda S, Aoki N. All-trans retinoic acid upregulates thrombomodulin and downregulates tissue-factor expression in acute promyelocytic leukemia cells: distinct expression of thrombomodulin and tissue factor in human leukemic cells. Blood 1994;84(09):3001-3009

33 Saito T, Koyama T, Nagata K, Kamiyama R, Hirosawa S. Anticoagulant effects of retinoic acids on leukemia cells. Blood 1996;87(02): 657-665

34 de la Serna J, Montesinos P, Vellenga E, et al. Causes and prognostic factors of remission induction failure in patients with acute promyelocytic leukemia treated with all-trans retinoic acid and idarubicin. Blood 2008;111(07):3395-3402
35 Lo-Coco F, Avvisati G, Vignetti M, et al; Gruppo Italiano Malattie Ematologiche dell'Adulto German-Austrian Acute Myeloid Leukemia Study Group Study Alliance Leukemia. Retinoic acid and arsenic trioxide for acute promyelocytic leukemia. N Engl J Med 2013;369(02):111-121

36 Burnett AK, Russell NH, Hills RK, et al; UK National Cancer Research Institute Acute Myeloid Leukaemia Working Group. Arsenic trioxide and all-trans retinoic acid treatment for acute promyelocytic leukaemia in all risk groups (AML17): results of a randomised, controlled, phase 3 trial. Lancet Oncol 2015;16(13): 1295-1305

37 Sanz MA, Fenaux P, Tallman MS, et al. Management of acute promyelocytic leukemia: updated recommendations from an expert panel of the European LeukemiaNet. Blood 2019;133 (15):1630-1643

38 Rodeghiero F, Avvisati G, Castaman G, Barbui T, Mandelli F. Early deaths and anti-hemorrhagic treatments in acute promyelocytic leukemia. A GIMEMA retrospective study in 268 consecutive patients. Blood 1990;75(11):2112-2117

39 Cao M, Li T, He Z, et al. Promyelocytic extracellular chromatin exacerbates coagulation and fibrinolysis in acute promyelocytic leukemia. Blood 2017;129(13):1855-1864

40 Leader A, Ten Cate H, Spectre G, Beckers EAM, Falanga A. Antithrombotic medication in cancer-associated thrombocytopenia: current evidence and knowledge gaps. Crit Rev Oncol Hematol 2018;132:76-88

41 Feldman EJ, Arlin ZA, Ahmed T, et al. Acute promyelocytic leukemia: a 5-year experience with new antileukemic agents and a new approach to preventing fatal hemorrhage. Acta Haematol 1989;82 (03):117-121

42 Hambley BC, Norsworthy KJ, Jasem J, et al. Fibrinogen consumption and use of heparin are risk factors for delayed bleeding during acute promyelocytic leukemia induction. Leuk Res 2019; 83:106174

43 Watanabe-Kusunoki K, Nakazawa D, Ishizu A, Atsumi T. Thrombomodulin as a physiological modulator of intravascular injury. Front Immunol 2020;11:575890

44 Matsushita T, Watanabe J, Honda G, et al. Thrombomodulin alfa treatment in patients with acute promyelocytic leukemia and disseminated intravascular coagulation: a retrospective analysis of an open-label, multicenter, post-marketing surveillance study cohort. Thromb Res 2014;133(05):772-781

45 Martí-Carvajal AJ, Anand V, Solà I Treatment for disseminated intravascular coagulation in patients with acute and chronic leukemia. Cochrane Database Syst Rev 2015;2015(06):CD008562

46 Fredenburgh JC, Weitz JI. New anticoagulants: moving beyond the direct oral anticoagulants. J Thromb Haemost 2021;19(01):20-29

47 Visser M, Heitmeier S, Ten Cate H, Spronk HMH. Role of factor XIa and plasma kallikrein in arterial and venous thrombosis. Thromb Haemost 2020;120(06):883-993 